\title{
A corrector for the Sverdrup solution for a domain with islands
}

\author{
D. Bresch`, F. Guillén-Gonzalez ••, M.A. Rodríguez-Bellido••
}

- Laboratoire de Mathématiques Appliquées (UMR6620), Université

Blaise Pascal, 63177 Aubière cedex, France.

e-mail: Didier.Bresch@math.univ-bpclermont.fr

•• Dpto. de Ecuaciones Diferenciales y Análisis Numérico,

Universidad de Sevilla,

Aptdo. 1160, 41080 Sevilla, Espagne.

e-mail: guillen@numer.us.es, angeles@numer.us.es

\begin{abstract}
In this paper we look at the influence of the Coriolis force on the quasi-geostrophic equations on a domain with islands. We prove that asymptotically we obtain the solution of the Sverdrup equation with homogeneous Dirichlet conditions on the inward boundary plus a corrector function which takes into account the presence of the islands. This work is motivated by the fact that in oceanography most of the surfaces are not simply connected. This is the case for example for the North Pacific with the Japanese islands. At our knowledge, in all the previous mathematical works, just simply connected domains have been considered. Finally we will give some simple numerical simulations related to the Stommel model to see the importance of the corrector.
\end{abstract}

Keywords. Ocean circulation, asymptotic model, singular perturbations, islands.

AMS subjects classification. 35Q30, 35B40, 76D05. 


\section{Introduction}

We consider $\Omega \subset \mathbb{R}^{2}$ the surface of a water extension that has some islands, which is the case of North Pacific Ocean with the Japanese islands, for instance. All the models used to obtain the Sverdrup relation through an asymptotic analysis consider the case of a simply connected domain, which implies Dirichlet homogeneous boundary conditions in the initial model. Here, we consider the case of a bidimensional domain $\Omega$ with an island. The case of several islands can be treated in the same way. A simple model, called the quasi-geostrophic equation with one layer (of constant depth), allows us to describe roughly the stream intensification on the West coasts. We consider the case of an island, that means the domain of figure 1 given by $\Omega=\Omega_{1} \backslash \bar{\Omega}_{2}$ with $\Omega_{i} \subset \mathbb{R}^{2}$ simply connected, $\Omega_{2} \subset \subset \Omega_{1}$, and $\Gamma_{i}=\partial \Omega_{i}$. The model related to the stream function $\Psi$ can be described as:

$$
\left\{\begin{array}{l}
E \Delta^{2} \Psi-\mu \Delta \Psi+\varepsilon \nabla^{\perp} \Psi \cdot \nabla \Delta \Psi+a \cdot \nabla \Psi=\nabla^{\perp} \cdot f \text { in } \Omega \\
\Psi=0 \text { on } \Gamma_{1} \\
\Psi=c_{E, \mu, \varepsilon} \text { on } \Gamma_{2} \\
\nabla \Psi \cdot n=0 \text { on } \partial \Omega
\end{array}\right.
$$

with the compatibility condition

$$
\int_{\Gamma_{2}}\left(E \nabla(\Delta \Psi)+f^{\perp}\right) \cdot n=0
$$

where $E, \mu$ y $\varepsilon$ are small positive constants, $\nabla^{\perp}=\left(-\partial_{y}, \partial_{x}\right), a=$ $(-1,0), f^{\perp}=\left(-f_{2}, f_{1}\right)$ and $n$ is the exterior unit normal of the boundary $\partial \Omega$. We remark that $\nabla^{\perp}$. corresponds to the curl operator.

To get this compatibility condition, we use

$$
\int_{\Gamma_{2}} \nabla \Psi \cdot n=\int_{\Gamma_{2}} a \cdot n=\int_{\Gamma_{2}} \Delta \Psi \nabla^{\perp} \Psi \cdot n=0 .
$$

First and last equalities are obtained thanks to the boundary conditions (in particular $\nabla \Psi=0$ on $\partial \Omega$, since $\nabla \Psi \cdot \tau=\nabla \Psi \cdot n=0$ on $\partial \Omega$ ), and the second one due to the fact that $\Gamma_{2}$ is a closed curve (is a Jordan curve or a simple closed curve). The reader interested by compatibility conditions on fluid mechanics problems is referred for instance to [7] and references cited therein. 
We note that such model is used to describe vertically averaged flows in a three dimensional flat domain in terms of the stream function $\Psi$ associated to the mean velocity field $u=\left(-\partial_{y} \Psi, \partial_{x} \Psi\right)$.

The purpose of this work is to perform the asymptotic analysis when $E, \mu, \varepsilon$ converge to 0 . In a first step (Theorem 1 ), we assume that $\varepsilon=0$ (that is to say, the linear case). The study of a such linear equation is interesting from a pedagogical point of view, see for instance [9] and [12]. We obtain at the limit the Sverdrup solution with a corrector which takes into account the presence of the island. In a second step (Theorem 2) we show how to extend the result to the nonlinear case that means the case $\varepsilon \neq 0$. In the last section, we present a simple simulation on the Stommel model with an island. We see the influence of the island.

An asymptotic study on the same 4th order model in the nonlinear case $(\varepsilon \neq 0)$ was made in [2] and over a Stommel type model (2nd order for $E=\varepsilon=0$ ) in [3] for a field $a$ tangent to the boundary and which tends locally to a field $\bar{a}$ transversal to the boundary. The case of model (1) with $E=\varepsilon=0$, homogeneous boundary condition and $a=(-1,0)$ has been largely studied because there are many physical applications modelled by this kind of equation. All the previous works are only related to a simply connected domain $\Omega$.

We note that the configuration of the domain implies the presence of characteristic boundary layers (North, South), free boundary layers (issued from the south and the north of the island), Stommel or Munk layers (Western part of the domain). We did not study here the associated boundary layers correctors necessary to obtain better convergence results since we are only interested by the main order. This will be done in a forthcoming work related to the study of characteristic boundaries.

The non-stationary case in a simply connected domain is studied in [5] where they build the western boundary layers and they obtain an approximate solution. They assume that $\nabla^{\perp} \cdot f$ vanishes in a neightbourhood of the North and the South Parts of the boundary. It allows them to not study the characteristic boundary layers which appear for general data. Here we consider the stationary version of the quasigeostrophic equations. This may be seen as the study of the long time behavior of the flow. We have not the time derivative to obtain better convergence results as in [5]. 


\section{Internal convergence and close to East coasts.}

Here, we will prove the following result

Theorem 1 Let $\Omega$ be a domain of $\mathcal{C}^{2}$ class with $\partial \Omega=\Gamma_{1} \cup \Gamma_{2}$ where $\bar{\Gamma}_{1} \cap \bar{\Gamma}_{2}=\emptyset$ defined as in figure 1 . Let $f \in H^{1}(\Omega)^{2}$ such that $\nabla^{\perp} \cdot f \in$ $H^{2}(\Omega)$. Let $\Psi$ be a solution in $H^{4}(\Omega)$ of $(1)$ with $\varepsilon=0$ and $(2)$. For all neighbourhood $V=V_{-} \cup V_{I, I I}$ of $\Gamma^{-} \cup \Gamma_{I, I I}$, where $\Gamma^{-}=\{x \in \partial \Omega$ : $\left.n_{x} \leq 0\right\}$ and $\Gamma_{I, I I}=\overline{\Omega^{I}} \cap \overline{\Omega^{I I}}$, with $\Omega^{I}, \Omega^{I I}$ as in figure 2, one has

$$
\begin{gathered}
\Psi \rightarrow \bar{\Psi}+c 1_{\Omega^{I I}} \text { weakly in } L^{2}(\Omega) \text { and strongly in } L^{2}(\Omega \backslash V), \\
\partial_{x} \Psi \rightarrow \partial_{x} \bar{\Psi} \text { weakly in } L^{2}\left(\Omega \backslash V_{-}\right)
\end{gathered}
$$

where $\bar{\Psi}$ is the solution in $L^{2}(\Omega) \cap H^{2}\left(\Omega \backslash \Gamma_{I, I I}\right)$ of the Sverdrup equation

$$
\left\{\begin{array}{l}
-\partial_{x} \bar{\Psi}=\nabla^{\perp} \cdot f \text { in } \Omega \\
\bar{\Psi}=0 \text { on } \Gamma^{+}
\end{array}\right.
$$

and $c$ is computed by the equality

$$
c=\frac{-\int_{\Gamma_{2}^{-}} \bar{\Psi} n_{x}+\int_{\Gamma_{2}} f^{\perp} \cdot n}{\int_{\Gamma_{2}^{+}} n_{x}}
$$

with $\Gamma_{2}^{+}=\left\{x \in \Gamma_{2}: n_{x}>0\right\}, \Gamma_{2}^{-}=\left\{x \in \Gamma_{2}: n_{x}<0\right\}$ and $1_{\Omega^{I I}}$ the characteristic function of the sub-domain $\Omega^{I I}$.

Remark. We assume the same kind of regularity on $f$ than in [7] to obtain our result. More precisely, we assume $\nabla^{\perp} \cdot f \in H^{2}(\Omega)$ and they assume that $\nabla^{\perp} \cdot f \in W^{1, \infty}\left(0, T ; H^{2}(\Omega)\right)$.

\section{Proof.}

Existence. We use the linearity of the problem and the uniqueness of solution for the equation (1) with $\varepsilon=0$. In this way, we decompose the unknown of the problem (1), $\Psi$, into

$$
\Psi=\Psi_{1}+c_{E, \mu} \Psi_{2}
$$


where $\Psi_{1}$ and $\Psi_{2}$ are respectively strong solutions (in $H^{4}(\Omega)$ ) of

$$
\left\{\begin{array}{l}
E \Delta^{2} \Psi_{1}-\mu \Delta \Psi_{1}-\partial_{x} \Psi_{1}=\nabla^{\perp} \cdot f \text { in } \Omega \\
\Psi_{1}=0 \text { on } \partial \Omega \\
\nabla \Psi_{1} \cdot n=0 \text { on } \partial \Omega
\end{array}\right.
$$

and

$$
\left\{\begin{array}{l}
E \Delta^{2} \Psi_{2}-\mu \Delta \Psi_{2}-\partial_{x} \Psi_{2}=0 \text { in } \Omega \\
\Psi_{2}=0 \text { on } \Gamma_{1} \\
\Psi_{2}=1 \text { on } \Gamma_{2} . \\
\nabla \Psi_{2} \cdot n=0 \text { on } \partial \Omega
\end{array}\right.
$$

The existence and uniqueness of the solutions $\Psi_{1}$ and $\Psi_{2}$ in $H^{4}(\Omega)$ is a classic result, $c f$. [6]. The constant $c_{E, \mu}$ can be determined by the compatibility condition (2) as,

$$
c_{E, \mu}=-\frac{\int_{\Gamma_{2}}\left(E \nabla \Delta \Psi_{1}+f^{\perp}\right) \cdot n}{\int_{\Gamma_{2}} E \nabla \Delta \Psi_{2} \cdot n} .
$$

Remark that $\int_{\Gamma_{2}} E \nabla \Delta \Psi_{2} \cdot n \neq 0$, because if we multiply the equation for $\Psi_{2}$ by $\Psi_{2}$ and we integrate by parts, we obtain

$$
E \int_{\Omega}\left|\Delta \Psi_{2}\right|^{2}+\mu \int_{\Omega}\left|\nabla \Psi_{2}\right|^{2}+E \int_{\Gamma_{2}} \nabla \Delta \Psi_{2} \cdot n=0
$$

and this would imply that $\Psi_{2}=0$ if we impose that $\int_{\Gamma_{2}} E \nabla \Delta \Psi_{2} \cdot n=$ 0 .

Convergence. From expression (3), we have to observe the convergence for the different terms $\Psi_{1}, \Psi_{2}$ et $c_{E, \mu}$, when $E, \mu \rightarrow 0$. The convergence of $c_{E, \mu}$ needs to introduce a function $\theta$ because we will only know the weak convergence in $L^{2}(\Omega)$ for $\Psi_{1}$ and $\Psi_{2}$ in the whole $\Omega$. For the sake of simplicity, we will not remark the dependency from $E$ and $\mu$ in $\Psi_{1}$ and $\Psi_{2}$.

i) Convergence for $\Psi_{1}$. As $f \in H^{1}(\Omega), \nabla^{\perp} \cdot f \in H^{2}(\Omega)$ and $\Omega$ is given by figure 1, using the results obtained in [2] and [4], we get:

$$
\begin{gathered}
\Psi_{1} \rightarrow \bar{\Psi}_{1} \text { weakly in } L^{2}(\Omega) \text { and strongly in } L^{2}(\Omega \backslash V), \\
\partial_{x} \Psi_{1} \rightarrow \partial_{x} \bar{\Psi}_{1} \text { weakly in } L^{2}\left(\Omega \backslash V_{-}\right)
\end{gathered}
$$


where $\bar{\Psi}_{1}$ is the solution in $L^{2}(\Omega)$ for the following Sverdrup "homogeneous" problem:

$$
\left\{\begin{array}{l}
-\partial_{x} \bar{\Psi}_{1}=\nabla^{\perp} \cdot f \text { in } \Omega, \\
\bar{\Psi}_{1}=0 \text { on } \Gamma^{+} .
\end{array}\right.
$$

Existence and uniqueness of a solution for the previous problem is done in [1]. Remark that $\bar{\Psi}_{1}$ is smooth in $\Omega \backslash V$, more precisely $\bar{\Psi}_{1} \in$ $H^{2}(\Omega \backslash V)$.

We review quickly the main steps used in [2] for the reader's convenience. These steps allow to establish the convergence from $\Psi_{1}$ through $\bar{\Psi}_{1}$. Multiplying the equation verified by $\Psi_{1}$ by $\Psi_{1} e^{x}$, we obtain

$$
\left\|\Psi_{1}\right\|_{L^{2}(\Omega)} \leq C
$$

where $C$ is independent from $E$ and $\mu$. Then, multiplying by $\Psi_{1}$ we get the estimate

$$
E\left\|\Delta \Psi_{1}\right\|_{L^{2}(\Omega)}^{2}+\mu\left\|\nabla \Psi_{1}\right\|_{\left(L^{2}(\Omega)\right)^{2}}^{2} \leq C_{1}
$$

where $C_{1}$ is independent from $E$ and $\mu$. These estimates allow us to get the weak limit in $L^{2}$ for a subsequence that tends to a solution of the Sverdrup relation, that is to say, without boundary conditions.

To prove the weak convergence of $\partial_{x} \Psi_{1}$ through $\partial_{x} \overline{\Psi_{1}}$ in $L^{2}\left(\Omega \backslash V_{-}\right)$, we only have to test the equation satisfied by $\Psi_{1}$ against $\left(\partial_{x} \Psi_{1}\right) \eta$ where $\eta \in \mathcal{C}^{2}(\bar{\Omega}), \eta=0$ in $V_{-}$and $\eta \geq 0$ in $\Omega$. We obtain an uniform estimate for $\int_{\Omega}\left|\partial_{x} \Psi_{1}\right|^{2} \eta$. This gives the strong convergence of $\Psi_{1}$ in $L^{2}(\Omega \backslash V)$. The idea is the same as in [7] for a simply connected domain and the linear case, and as in [4] for the nonlinear case. This last argument, use the boundary condition $\nabla \Psi_{1} \cdot n=0$ sur $\Gamma_{+}$strongly.

ii) Convergence for $\Psi_{2}$. Now, we focus on the problem for $\Psi_{2}$. First, we lift the boundary condition to study an homogeneous problem as we heve done for $\Psi_{1}$. More concretely, we consider $\xi \in \mathcal{C}^{4}(\bar{\Omega})$ such that $\xi=0$ on a neighbourhood of $\Gamma_{1}$ and $\xi=1$ on a neighbourhood of $\Gamma_{2}$. If we take $\Psi_{2}=\widetilde{\Psi}_{2}+\xi$ then $\widetilde{\Psi}_{2}$ verifies

$$
\left\{\begin{array}{l}
E \Delta^{2} \widetilde{\Psi}_{2}-\mu \Delta \widetilde{\Psi}_{2}-\partial_{x} \widetilde{\Psi}_{2}=-E \Delta^{2} \xi+\mu \Delta \xi+\partial_{x} \xi \text { in } \Omega \\
\widetilde{\Psi}_{2}=0 \text { on } \partial \Omega \\
\nabla \widetilde{\Psi}_{2} \cdot n=0 \text { on } \partial \Omega
\end{array}\right.
$$


The reasoning will finish in the same way that for $\Psi_{1}$, i.e.

$$
\begin{gathered}
\widetilde{\Psi}_{2} \rightarrow \overline{\widetilde{\Psi}_{2}} \text { weakly in } L^{2}(\Omega) \text { and strongly in } L^{2}(\Omega \backslash V), \\
\partial_{x} \widetilde{\Psi}_{2} \rightarrow \partial_{x} \overline{\widetilde{\Psi}_{2}} \text { weakly in } L^{2}\left(\Omega \backslash V_{-}\right)
\end{gathered}
$$

where $\overline{\widetilde{\Psi}_{2}}$ is the solution in $L^{2}(\Omega)$ of :

$$
\left\{\begin{array}{l}
-\partial_{x} \overline{\widetilde{\Psi}_{2}}=\partial_{x} \xi \text { in } \Omega, \\
\overline{\widetilde{\Psi}_{2}}=0 \text { on } \Gamma^{+} .
\end{array}\right.
$$

Therefore $\Psi_{2} \rightarrow \overline{\Psi_{2}}=\overline{\widetilde{\Psi}_{2}}+\xi$ weakly in $L^{2}(\Omega)$, strongly in $L^{2}(\Omega \backslash V)$ and $\partial_{x} \Psi_{2} \rightarrow \partial_{x} \overline{\Psi_{2}}$ weakly in $L^{2}\left(\Omega \backslash V_{-}\right)$where $\bar{\Psi}_{2}$ is the solution in $L^{2}(\Omega)$ of

$$
\left\{\begin{array}{l}
-\partial_{x} \bar{\Psi}_{2}=0 \text { in } \Omega \\
\bar{\Psi}_{2}=0 \text { on } \Gamma_{1}^{+} \\
\bar{\Psi}_{2}=1 \text { on } \Gamma_{2}^{+}
\end{array}\right.
$$

that is to say $\bar{\Psi}_{2}=1_{\Omega^{I I}}$.

The characteristic line crossing trough the extremal points of an island divide the domain in two subregions denoted as $\Omega^{I}$ and $\Omega^{I I}$. Then it appears a boundary layer along $\Gamma_{I, I I}$.

iii) Convergence for the constant $c_{E, \mu}$. Let $\theta \in H^{2}(\Omega)$ be such that $\theta=0$ on a neighbourhood of $\Gamma_{1}$ and $\theta=1$ on a neighbourhood of $\Gamma_{2}$. From the equation verified by $\Psi_{1}$, we obtain

$$
\begin{aligned}
\nabla \cdot\left(E \theta \nabla \Delta \Psi_{1}-\mu \theta \nabla \Psi_{1}+\theta a \Psi_{1}+\theta f^{\perp}\right) & =E \nabla \theta \cdot \nabla \Delta \Psi_{1} \\
& -\mu \nabla \theta \cdot \nabla \Psi_{1}+\nabla \theta \cdot a \Psi_{1}+\nabla \theta \cdot f^{\perp}
\end{aligned}
$$

and $a=(-1,0)$. Then, integrating in $\Omega$, and using that $\nabla \theta=0$ on $\Gamma_{1} \cup \Gamma_{2}, \nabla \Psi_{1} \cdot n=0$ on $\Gamma$, we get

$$
\begin{aligned}
\int_{\Gamma_{2}} E \nabla \Delta \Psi_{1} \cdot n & +f^{\perp} \cdot n=-\int_{\Omega} E \Delta \theta \Delta \Psi_{1} \\
& +\mu \int_{\Omega} \nabla \theta \cdot \nabla \Psi_{1}+\int_{\Omega} \nabla \theta \cdot a \Psi_{1}+\int_{\Omega} \nabla \theta \cdot f^{\perp} .
\end{aligned}
$$

We saw that $\left\|\Psi_{1}\right\|_{L^{2}(\Omega)} \leq C, E\left\|\Delta \Psi_{1}\right\|_{L^{2}(\Omega)}^{2} \leq C, \mu\left\|\nabla \Psi_{1}\right\|_{L^{2}(\Omega)}^{2} \leq C$ with $C$ independent from $E$ and $\mu$. Therefore, for $E, \mu \rightarrow 0$ 


$$
\begin{aligned}
\int_{\Gamma_{2}} E \nabla \Delta \Psi_{1} \cdot n+f^{\perp} \cdot n \rightarrow & \int_{\Omega} \nabla \theta \cdot a \bar{\Psi}_{1}+\int_{\Omega} \nabla \theta \cdot f^{\perp} \\
& \left(=\int_{\Gamma_{2}^{-}} \bar{\Psi}_{1} n_{x}+\int_{\Gamma_{2}} f^{\perp} \cdot n\right) .
\end{aligned}
$$

Moreover, from the equation verified by $\Psi_{2}$,

$\nabla \cdot\left(E \theta \nabla \Delta \Psi_{2}-\mu \theta \nabla \Psi_{2}+\theta a \Psi_{2}\right)=E \nabla \theta \cdot \nabla \Delta \Psi_{2}-\mu \nabla \theta \cdot \nabla \Psi_{2}+\nabla \theta \cdot a \Psi_{2}$

thereby

$$
\int_{\Gamma_{2}} E \nabla \Delta \Psi_{2} \cdot n=-\int_{\Omega} E \Delta \theta \Delta \Psi_{2}+\mu \int_{\Omega} \nabla \theta \cdot \nabla \Psi_{2}+\int_{\Omega} \nabla \theta \cdot a \Psi_{2} .
$$

The limit for $\Psi_{2}$ is made as before for $\Psi_{1}$, obtaining

$$
\int_{\Gamma_{2}} E \nabla \Delta \Psi_{2} \cdot n \rightarrow-\int_{\Omega} \bar{\Psi}_{2} \partial_{x} \theta\left(\text { i.e. } \int_{\Omega} \nabla \theta \cdot a \bar{\Psi}_{2}\right) .
$$

But

$$
\int_{\Omega} \bar{\Psi}_{2} \partial_{x} \theta=\int_{\Omega_{I I}} \partial_{x} \theta=\int_{\Gamma_{2}^{+}} n_{x}
$$

Then,

$$
c_{E, \mu} \rightarrow-\frac{\int_{\Omega} \nabla \theta \cdot a \bar{\Psi}_{1}+\nabla \theta \cdot f^{\perp}}{\int_{\Gamma_{2}^{+}} n_{x}}:=c
$$

Therefore, $\Psi=\Psi_{1}+c_{E, \mu} \Psi_{2} \rightarrow \bar{\Psi}_{1}+c 1_{\Omega_{I I}}$ with $c$ given as before. Observe that $c$ does not depend on the function $\theta$ because it is the limit for $c_{E, \mu}$ that is independent from $\theta$. Let us rewrite it in another form. If we integrate by parts the numerator of the constant $c$ and if we use the Sverdrup equation satisfied by $\bar{\Psi}$ and the properties of $\theta$, we find

$$
c=\frac{-\int_{\Gamma_{2}^{-}} \bar{\Psi} n_{x}+\int_{\Gamma_{2}} f^{\perp} \cdot n}{\int_{\Gamma_{2}^{+}} n_{x}} .
$$

Finally, collecting all the previous results, we finish the proof of Theorem 1.

Remark. If we assume to have no tangential force on the boundary of the Island that means $f^{\perp} \cdot n=0$, we find exactly the constant $\Psi_{l}$ 
defined, Equality (2.8), in [10]. That means we find the vertical average value of the Sverdrup streamfunction on the eastern side of the island

$$
c=\frac{1}{\left(y_{n}-y_{s}\right)} \int_{y_{s}}^{y_{n}} \bar{\Psi}\left(x_{+}(y), y\right) d y
$$

where $x_{+}$denotes the graph of the eastern part of the Island, $y_{s}$ and $y_{n}$ are respectively the mimimum vertical coordinate (South), the maximum vertical coordinate (North) on the boundary of the island.

\section{Another boundary conditions}

It is possible to choose another boundary conditions different to $\nabla \Psi$. $n=0$ on $\partial \Omega$. We refer to [7] for the reader interested in a physical discussion on the possible boundary conditions for the quasi-geostrophic equations (1).

For the problem (1), changing for instance the boundary condition $\nabla \Psi \cdot n=0$ on $\partial \Omega$ by $\Delta \Psi=0$ on $\partial \Omega$ and conserving the Dirichlet type condition on $\Psi$, we will obtain essentially the same results of Theorem 1 (except the weak convergence in $L^{2}\left(\Omega \backslash V_{-}\right)$from $\partial_{x} \Psi$ to $\partial_{x} \bar{\Psi}$ that, seemingly, only works if $\nabla \Psi \cdot n=0$ on $\left.\Gamma^{+}\right)$.

To obtain the strong convergence in $L^{2}(\Omega \backslash V)$ through the solution $\bar{\Psi}_{1}$ in $L^{2}(\Omega)$ that vanishes on $\Gamma^{+}$, we only have to take the difference between the equation for $\Psi_{1}$ and the equation that verifies the Sverdrup solution $\bar{\Psi}_{1}$ that vanishes on $\Gamma^{+}$. Then test the resulting equation with $\left(\Psi_{1}-\bar{\Psi}_{1}\right) \Phi$, where $\Phi$ is given by

$$
\Phi(x, y)=\int_{g_{\mathrm{West}}(y)}^{x} \eta\left(x_{\star}, y\right) d x_{\star}
$$

for $\eta \in \mathcal{C}^{2}(\bar{\Omega}), \eta=0$ in $V$ and $\eta \geq 0$ in $\Omega$. Recall that we consider $g_{\text {East }}$ of $\mathcal{C}^{2}$ class.

In this case, we change the compatibility condition (2) by :

$$
\int_{\Gamma_{2}}\left(E \nabla \Delta \Psi-\mu \nabla \Psi+f^{\perp}\right) \cdot n=0 .
$$

Accordingly, if we consider the problem (1) with $\varepsilon=0$ and (7) we also obtain existence and uniqueness for a solution $\Psi$ in $H^{4}(\Omega)$. This solution is constructed as for (3) replacing the boundary conditions

$$
\nabla \Psi_{1} \cdot n=\nabla \Psi_{2} \cdot n=0 \text { on } \partial \Omega
$$


by

$$
\Delta \Psi_{1}=\Delta \Psi_{2}=0 \text { on } \partial \Omega,
$$

and the expression for the constant $c_{E, \mu}$ given in (4) by

$$
c_{E, \mu}=-\frac{\int_{\Gamma_{2}}\left(E \nabla \Delta \Psi_{1}-\mu \nabla \Psi_{1}+f^{\perp}\right) \cdot n}{\int_{\Gamma_{2}}\left(E \nabla \Delta \Psi_{2}-\mu \nabla \Psi_{2}\right) \cdot n} .
$$

Observe that $\int_{\Gamma_{2}}\left(E \nabla \Delta \Psi_{2}-\mu \nabla \Psi_{2}\right) \cdot n \neq 0$ because if we consider $\Psi_{2}$ as a function test for the problem for $\Psi_{2}$, then we get

$$
E \int_{\Omega}\left|\Delta \Psi_{2}\right|^{2}+\mu \int_{\Omega}\left|\nabla \Psi_{2}\right|^{2}+\int_{\Gamma_{2}}\left(E \nabla \Delta \Psi_{2}-\mu \nabla \Psi_{2}\right) \cdot n=0 .
$$

Therefore, we obtain the same kind of convergence than before because of the results of [2] are also proved for this type of boundary conditions. And then, we obtain the same limit function on $\Psi_{1}$ and $\Psi_{2}$ given by $\bar{\Psi}_{1}$ the Sverdrup solution and $\bar{\Psi}_{2}=1_{\Omega^{I I}}$.

Finally, we prove the convergence for the constant $c_{E, \mu}$ to the same limit of the previous section, $c$, adding to the left hand side of (5) and (6) respectively the integrals

$$
-\int_{\Gamma_{2}} \mu \nabla \Psi_{1} \cdot n \quad \text { and } \quad-\int_{\Gamma_{2}} \mu \nabla \Psi_{2} \cdot n
$$

Remark that the result is true even if we consider a combination of the two boundary conditions $\nabla \Psi \cdot n=0$ on $\Gamma$ and $\Delta \Psi=0$ on $\partial \Omega \backslash \bar{\Gamma}$. In this case, thanks to the regularity $H^{2}(\Omega) \cap H_{\text {loc }}^{3}(\Omega)$ for $\Psi$, we can easily determine the limit of the constant $c_{E, \mu}$.

This section allows us to state that the choice of the boundary conditions does not have any influence on the asymptotic stream in the interior and the East coasts. The influence of the second boundary condition can be remarked at the boundary layer level.

\section{Non linear case.}

We will prove by a comparison argument between the linear problem and the nonlinear one that for some range of the coefficients $E, \mu$ and $\varepsilon$, Theorem 1 can be extended to the nonlinear case that means $\varepsilon \neq 0$.

More precisely we prove the following result 
Theorem 2 Let $f$ be a function such that $f \in L^{2}(\Omega)$. Let $\Psi_{\varepsilon}$ be a solution of the quasi-geostrophic equation with $\varepsilon \neq 0$ and $\Psi$ the solution for $\varepsilon=0$. Then we get

$$
\left\|\Psi_{\varepsilon}-\Psi\right\|_{H^{1}(\Omega)} \leq C \frac{\varepsilon}{\mu^{5 / 4} E^{7 / 4}}
$$

with $C$ independent on $\varepsilon, E$ and $\mu$.

This result allow then to prove the same convergence than in Theorem 1 if $\varepsilon /\left(\mu^{5 / 4} E^{7 / 4}\right) \rightarrow 0$. It suffices to subtract then and to sum the solution corresponding to the linear problem.

\section{Proof.}

Let us consider the difference of the two solutions $\widetilde{\Psi}=\Psi_{\varepsilon}-\Psi$. It satisfies

$$
\left\{\begin{array}{l}
E \Delta^{2} \widetilde{\Psi}-\mu \Delta \widetilde{\Psi}+\varepsilon \nabla^{\perp} \Psi_{\varepsilon} \cdot \nabla \Delta \Psi_{\varepsilon}-\partial_{x} \widetilde{\Psi}=0 \text { in } \Omega \\
\widetilde{\Psi}=0 \text { on } \Gamma_{1} \\
\widetilde{\Psi}=c_{\varepsilon}-c \text { on } \Gamma_{2} \\
\nabla \widetilde{\Psi} \cdot n=0(\text { or } \Delta \widetilde{\Psi}=0) \partial \Omega .
\end{array}\right.
$$

Multiplying these equation by $\widetilde{\Psi}$, integrating by parts and using the compatibility condition valuable for $\widetilde{\Psi}$ and using the identity,

$$
\int_{\Omega} \nabla^{\perp} \Psi_{\varepsilon} \cdot \nabla \Delta \Psi_{\varepsilon} \widetilde{\Psi}=-\int_{\Omega} \nabla^{\perp} \Psi_{\varepsilon} \cdot \nabla \widetilde{\Psi} \Delta \Psi_{\varepsilon}
$$

we get

$$
E\|\Delta \widetilde{\Psi}\|_{L^{2}(\Omega)}^{2}+\mu\|\nabla \widetilde{\Psi}\|_{\left(L^{2}(\Omega)\right)^{2}}^{2}=\varepsilon \int_{\Omega} \nabla^{\perp} \Psi_{\varepsilon} \cdot \nabla \widetilde{\Psi} \Delta \Psi_{\varepsilon} .
$$

Let us remark that

$$
\begin{aligned}
\left|\int_{\Omega} \nabla^{\perp} \Psi_{\varepsilon} \cdot \nabla \widetilde{\Psi} \Delta \Psi_{\varepsilon}\right| & \leq\left\|\nabla \Psi_{\varepsilon}\right\|_{\left(L^{4}(\Omega)\right)^{2}}\|\nabla \widetilde{\Psi}\|_{\left(L^{4}(\Omega)\right)^{2}}\left\|\Delta \Psi_{\varepsilon}\right\|_{L^{2}(\Omega)} \\
& \leq C\left\|\nabla \Psi_{\varepsilon}\right\|_{\left(L^{2}(\Omega)\right)^{2}}^{1 / 2}\left\|\Delta \Psi_{\varepsilon}\right\|_{L^{2}(\Omega)}^{3 / 2}\|\nabla \widetilde{\Psi}\|_{\left(L^{2}(\Omega)\right)^{2}}^{1 / 2}\|\Delta \widetilde{\Psi}\|_{L^{2}(\Omega)}^{1 / 2} .
\end{aligned}
$$

Multiplying the nonlinear equation on $\Psi_{\varepsilon}$ by $\Psi_{\varepsilon}$, we get using the compatibility condition

$$
E\left\|\Delta \Psi_{\varepsilon}\right\|_{L^{2}(\Omega)}^{2}+\mu\left\|\nabla \Psi_{\varepsilon}\right\|_{L^{2}(\Omega)}^{2} \leq\|f\|_{L^{2}(\Omega)}\left\|\nabla \Psi_{\varepsilon}\right\|_{L^{2}(\Omega)} .
$$


Then,

$$
E \mu\left\|\Delta \Psi_{\varepsilon}\right\|_{L^{2}(\Omega)}^{2}+\mu^{2}\left\|\nabla \Psi_{\varepsilon}\right\|_{\left(L^{2}(\Omega)\right)^{2}}^{2} \leq C_{1}
$$

and

$$
E^{2}\left\|\Delta \Psi_{\varepsilon}\right\|_{L^{2}(\Omega)}^{2}+\mu E\left\|\nabla \Psi_{\varepsilon}\right\|_{\left(L^{2}(\Omega)\right)^{2}}^{2} \leq C_{2}
$$

with $C_{1}$ and $C_{2}$ independent on $\varepsilon, E, \mu$. In the second inequality, we have used that $\left\|\nabla \Psi_{\varepsilon}\right\|_{L^{2}(\Omega)} \leq K\left\|\Delta \Psi_{\varepsilon}\right\|_{L^{2}(\Omega)}$, with $K>0$ independent on $\varepsilon$. This is a consequence of the $H^{2}$ regularity of the Neumann Poisson problem when $\nabla \Psi_{\varepsilon} \cdot n=0$ is imposed on $\partial \Omega$. For other boundary conditions, we can use, taking $a=\nabla \Psi_{\varepsilon}$, that $\|a\|_{H^{1}(\Omega)} \leq$ $K\left(\|\nabla \cdot a\|_{L^{2}(\Omega)}+\left\|\nabla^{\perp} \cdot a\right\|_{L^{2}(\Omega)}\right), \forall a \in H^{1}$ such that $\int_{\Omega} a=0$ (see [6]).

Thus, using these two previous estimates, we get

$$
\begin{aligned}
\varepsilon\left|\int_{\Omega} \nabla^{\perp} \Psi_{\varepsilon} \cdot \nabla \widetilde{\Psi} \Delta \Psi_{\varepsilon}\right| \leq \frac{E}{2} & \|\Delta \widetilde{\Psi}\|_{L^{2}(\Omega)}^{2} \\
& +\frac{\mu}{2}\|\nabla \widetilde{\Psi}\|_{\left(L^{2}(\Omega)\right)^{2}}^{2}+C \frac{\varepsilon^{2}}{\mu^{3 / 2} E^{7 / 2}} .
\end{aligned}
$$

with $C$ independent on $\varepsilon, E, \mu$. Then using (9) and (10) and Poincare inequality, we get the conclusion.

Notice that there are more posibilities for the estimation done in (10). Indeed, one can change $\varepsilon^{2} /\left(\mu^{3 / 2} E^{7 / 2}\right)$ by $\varepsilon^{2} /\left(\mu^{3} E^{2}\right)$ or $\varepsilon^{2} /\left(\mu E^{4}\right)$ or $\varepsilon^{2} /\left(\mu^{5 / 2} E^{5 / 2}\right)$. Therefore, the condition $\varepsilon /\left(\mu^{5 / 4} E^{7 / 4}\right) \rightarrow 0$ for the convergence can be respectively changed by $\varepsilon /\left(\mu^{2} E\right) \rightarrow 0$ or $\varepsilon /\left(\mu E^{2}\right) \rightarrow$ 0 or $\varepsilon /\left(\mu^{7 / 4} E^{5 / 4}\right) \rightarrow 0$.

\section{Simulation for the Stommel model}

In this section, we consider the approximation from the Stommel model $(E=\varepsilon=0)$ through the Sverdrup equation, and we observe from a numerical point of view the influence of one island over the flux stream on the West coasts. We recommend [8] and also [12] for the reader interested in the Stommel relation without island. In any case, we recall the origin and meaning of the Stommel relation coefficients.

In 1948, Stommel proposed the following adimensional equation in 
a simply connected domain to describe the ocean circulation:

$$
\left\{\begin{array}{l}
-\frac{R}{\beta} \Delta \Psi-\partial_{x} \Psi=\nabla^{\perp} \cdot f \text { in } \Omega \\
\Psi=0 \text { on } \partial \Omega .
\end{array}\right.
$$

Here, $R=\omega_{0} d / 2 H L$ where $\omega_{0}=810^{-5} \mathrm{~s}^{-1}$ is the Earth rotating velocity, $H=3000 \mathrm{~m}$ is the characteristic depth, $L=1000 \mathrm{~km}$ is the characteristic length and $d=\left(2 \nu / \omega_{0}\right)^{1 / 2}$ is the characteristic depth for the Ekman layer, where $\nu=10^{-2} \mathrm{~m}^{2} \mathrm{~s}^{-1}$ is the turbulent viscosity, then $d \approx 16 \mathrm{~m}$ and $R \approx 2.110^{-13} \mathrm{~m}^{-1} \mathrm{~s}^{-1}$. On the other hand, $\beta=1.910^{-11}$ $\mathrm{m}^{-1} \mathrm{~s}^{-1}$ is the coefficient correspondent to the $\beta$-plan approximation. Consequently, $R / \beta \approx 10^{-2}$.

With one island (see domain $\Omega$ defined in figure 1 ), Stommel equation can be written as:

$$
\left\{\begin{array}{l}
-\mu \Delta \Psi+a \cdot \nabla \Psi=\nabla^{\perp} \cdot f \text { in } \Omega \\
\Psi=0 \text { on } \Gamma_{1} \\
\Psi=c_{\mu} \text { on } \Gamma_{2}
\end{array}\right.
$$

with $a=(-1,0), \mu=R / \beta$ and with the compatibility condition

$$
\int_{\Gamma_{2}}\left(-\mu \nabla \Psi+f^{\perp}\right) \cdot n=0
$$

If $\nabla^{\perp} \cdot f \in H^{1}(\Omega)$, we have existence and uniqueness of a solution $\Psi \in H^{2}(\Omega)$ for this system and we also have the asymptotic behaviour when $\mu \rightarrow 0$ through the same solution found in the previous sections. The solution $\Psi$ can be written as $\Psi=\Psi_{1}+c_{\mu} \Psi_{2}$ where $\Psi_{1}$ and $\Psi_{2}$ are the solutions of

$$
\left\{\begin{array}{l}
-\mu \Delta \Psi_{1}-\partial_{x} \Psi_{1}=\nabla^{\perp} \cdot f \text { in } \Omega, \\
\Psi_{1}=0 \text { on } \partial \Omega
\end{array}\right.
$$

and

$$
\left\{\begin{array}{l}
-\mu \Delta \Psi_{2}-\partial_{x} \Psi_{2}=0 \text { in } \Omega \\
\Psi_{2}=0 \text { on } \Gamma_{1} \\
\Psi_{2}=1 \text { on } \Gamma_{2}
\end{array}\right.
$$


respectively, with $c_{\mu}$ given by

$$
c_{\mu}=-\frac{\int_{\Gamma_{2}}\left(\mu \nabla \Psi_{1}-f^{\perp}\right) \cdot n}{\int_{\Gamma_{2}}\left(\mu \nabla \Psi_{2}\right) \cdot n} .
$$

We will use numerically the expression for $c_{\mu}$ on all $\Omega$ obtained due to $\theta$ as in the previous sections. More concretely, we will use

$$
c_{\mu}=\frac{\mu \int_{\Omega} \nabla \theta \nabla \Psi_{1}-\int_{\Omega} \nabla \theta \cdot a \Psi_{1}-\int_{\Omega} \nabla \theta \cdot f^{\perp}}{\mu \int_{\Omega} \nabla \theta \nabla \Psi_{2}-\int_{\Omega} a \cdot \nabla \theta \Psi_{2}} .
$$

Now we give some simulations on the Stommel model (12) and (14) with the data usually found in geophysical books. The interested reader is referred to [7] and [12].

We consider a square domain $\Omega=(0,1)^{2}$ with an ellipsoidal island and a wind of Munk type $f(x, y)=(\cos (\pi y) / \pi, 0)$. Then, $\nabla^{\perp} \cdot f=$ $\sin (\pi y)$. The stream function is calculated by the FEM program, of O. Pironneau, $c f$. [11].

Figure 3 represents the stream function corresponding to the Stommel model with $\mu=3 \cdot 10^{-2}$ when we take into account the constant $c_{\mu}$. Figure 4 represents the stream function when we do not take into account the constant $c_{\mu}$, i.e., we consider $\Psi_{1}$ only. The figure 5 shows the difference that means $c_{\mu} \Psi_{2}$.

This last figure shows the importance of this corrector function which converges asymptotically to $c 1_{\Omega^{I I}}$ in $\Omega \backslash V$.

Acknowledgments. The first author would like to thank Professor Alain Colin de verdière for interesting discussions on this paper. After the redaction of this paper, he indicates him the physical paper written by J. Pedlosky \&. The second and third authors have been partially supported by the C.I.C.Y.T. project MAR98-0486.

\section{References}

[1] C. Bardos. Problèmes aux limites pour les équations aux dérivées partielles du premier ordre à coefficients réels ; théorèmes d'approximation ; application à l'équation de transport. Ann. Scient. Éc. Norm. Sup., 3, (1970), 185-233. 
[2] D. Bresch, T. Colin. Some remarks on the derivation of the Sverdrup relation, to appear in J. Math. Fluid Mech, (2002).

[3] D. Bresch, J. Simon. On the western intensification of currents in Oceanic basins. Proceedings of II Workshop on Analysis of Variables and $\mathrm{Nu}$ merical Simulation of the Water Masses Exchanges through the Strait of Gibraltar, J. J. Alonso del Rosario and F. Ortegon Gallego (Eds), Universidad de Cádiz, (2000), 35-45.

[4] T. Colin. Remarks on a homogeneous model of ocean circulation. Asymptotic Anal., 12, (1996), 153-168.

[5] B. Desjardins, E. Grenier. On the homogeneous model of wind-driven ocean circulation. SIAM J. Appl. Math., 60, No. 1, (1999), 43-60.

[6] V. Girault, A. Raviart. Finite element approximation of the Navier-Stokes equations. Lecture Notes in Mathematics, Springer-Verlag, New-York (1981).

[7] J.-L. Lions. Perturbations singulières dans les problèmes aux limites et en contrôle optimal. Vol. 323, Springer-Verlag, Berlin, (1973).

[8] J. Pedlosky, Geophysical fluid dynamics. Springer-Verlag, (1987).

[9] J. Pedlosky, Ocean Circulation Theory. Springer-Verlag, (1996).

[10] J. Pedlosky, L.J. Pratt, M.A. Spall, K.R. Helrich. Circulation around Islands and ridges, J. Marine Research, 55, (1997), 1199-1251.

[11] O. Pironneau et al., Free FEM (software file). On the web, http://www.asci.fr.

[12] R. Salmon. Geophysical fluid dynamics. Oxford University Press, (1998). 


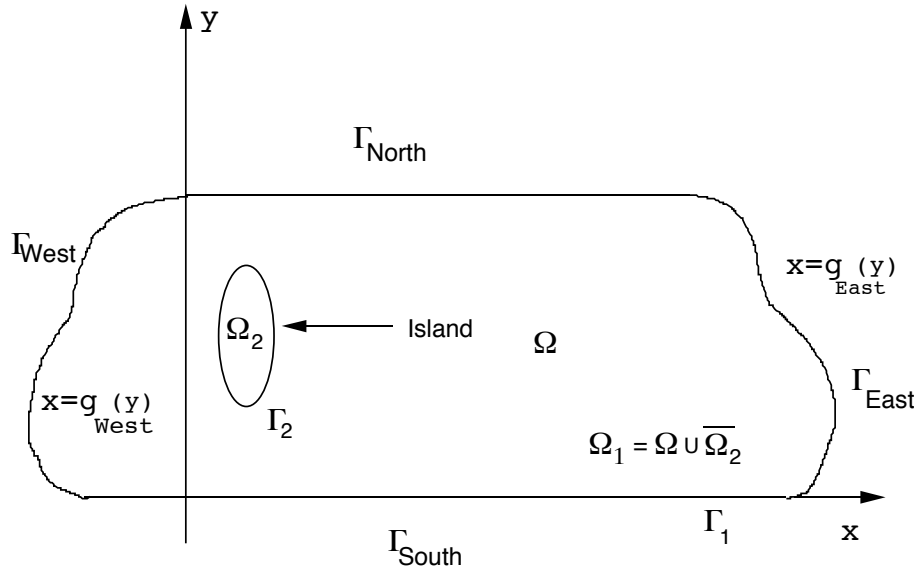

Fig. 1: The domain.

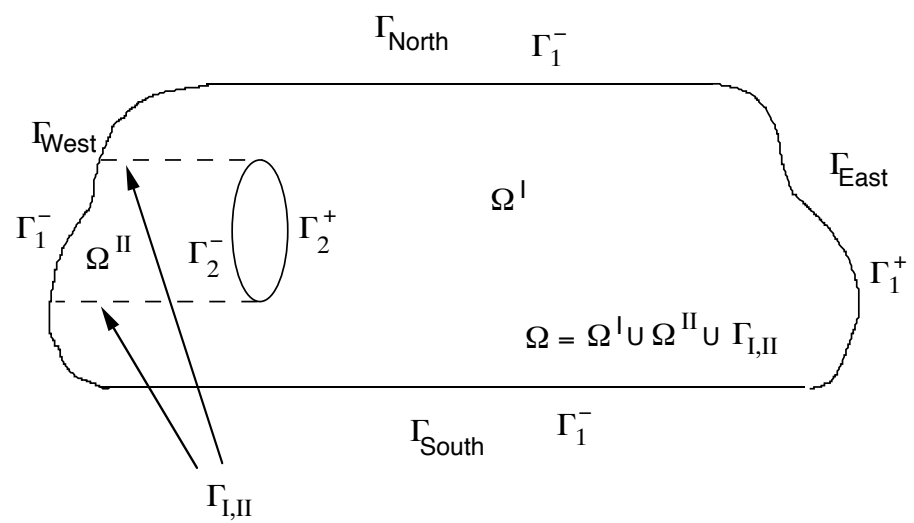

Fig. 2: The subdomains. 


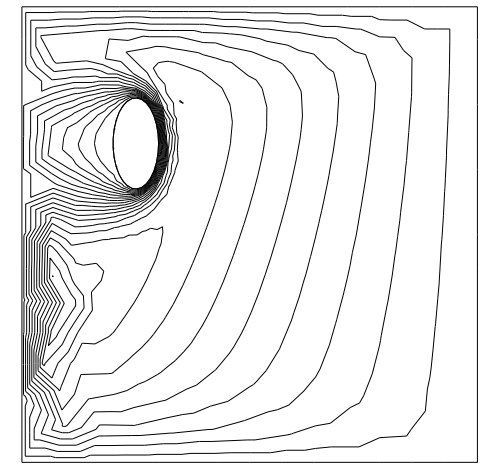

Fig. 3: The complete stream function.

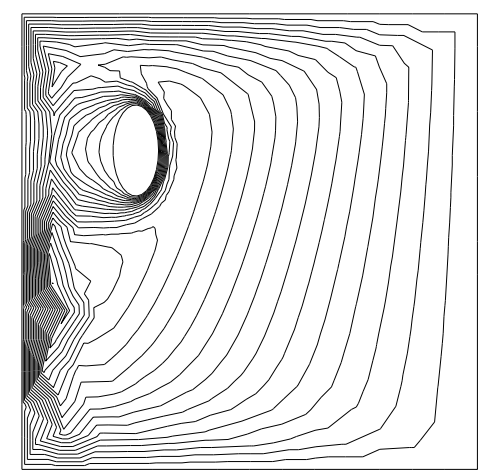

Fig. 4: The sverdrup solution. 


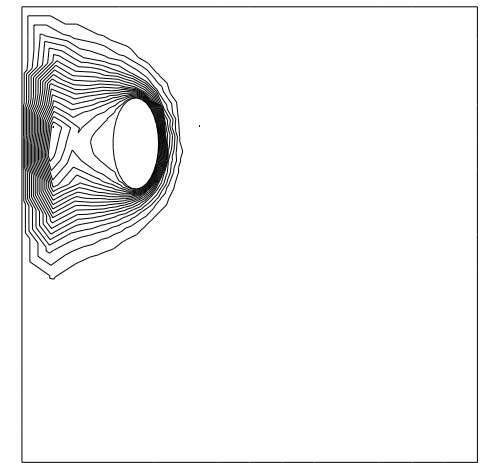

Fig. 5: The corrector. 